\title{
Portfolio und Mehrsprachigkeitsförderung
}

\author{
Michael Langner (Freiburg/Schweiv)
}

\section{Einleitung}

Porffolio und Mehrsprachigkeitsforderung - Ist der Titel nicht eigentlich schon ein Pleonasmus? Ist das Portfolio ${ }^{1}$ nicht das Instrument par excellence zur Förderung von Mehrsprachigkeit? Aber vielleicht rechtfertigt sich der Titel aus der Tatsache, dass in den vergangenen Jahren der Fokus der Arbeit mit Portfolios (oft ungerechtfertigter Weise) meist auf der Evaluation lag und weniger beim Dossier!

Aber es gibt ja auch andere Gründe für die Beschäftigung mit diesem Thema. Die derzeitige Situation der europäischen Mehrsprachigkeit ist lamentabel. Im Jahre 2002 wurde in Barcelona auf einem Treffen des Rates der Europäischen Union das sogenannte Barcelona-Prinzip formuliert: Alle Bürger/-innen der Europäischen Union sollten neben ihrer Muttersprache zwei Fremdsprachen beherrschen (L1 plus 2L). ${ }^{2}$ Davon sind wir derzeit weit entfernt, da die Anglifizierung des Alltags sehr schnell voranschreitet. Teilweise springen wir freiwillig auf diesen Zug auf, der positiv mit Internationalisierung, Globalisierung, Innovation etikettiert wird, aber durch die Ubiquität des Englischen dazu führt, dass Jugendliche nur schwer für

\footnotetext{
1 Wenn ich im Folgenden von Portfolio spreche, ist hier das Europäische Sprachenportfolio (ESP) gemeint. Es gibt eine Reihe anderer Portfolios und dies teilweise schon viel länger, und das ESP hat seinen Namen gerade von diesen anderen (Künstler-, Architekten-)Portfolios übernommen, in denen Beispiele von best practice in einem Dossier zusammengefasst werden.

2 Der Begriff Muttersprache ist zwar allgemein üblich, dennoch recht problematisch. Aus diesem Grund verwenden Fachleute den Begriff Erstsprache. Ebenso mag ich den Begriff beherrschen nicht und würde eher von effektiv verwenden, können, anwenden sprechen.
} 
eine weitere Fremdsprache zu motivieren sind (vgl. Hufeisen \& Neuner 2003: 9). In diesem Zusammenhang wird immer wieder vom Englischen als „Killersprache“ gesprochen. Dies ist keine Eigenschaft an sich des Englischen (oder irgendeiner anderen Sprache), sondern „Killersprache“ meint hier, dass eine Sprache, die international ein großes Renommée besitzt und eine beträchtliche Bedeutung hat, andere zu lernende Sprachen dominiert. Mit dem Erlernen einer solchen Sprache wird das Erlernen weiterer Sprachen für viele Menschen obsolet, was einer (europäischen) Mehrsprachigkeitspolitik diametral widerspricht. ${ }^{3}$ Genau hier müsste eine Mehrsprachigkeitsdidaktik greifen!

Wir können also festhalten, dass vor diesem Hintergrund gewandelter europäischer Gesellschaften eine (neue) Diskussion um den Einsatz des Portfolios zur Förderung eben dieser offiziell so sehr geschätzten Mehrsprachigkeit wichtig ist.

\section{Der Gemeinsame Europäische Referenzrahmen für Sprachen als Basis des Europäischen Sprachenportfolios}

Der Gemeinsame Europäische Referenærabmen für Sprachen (im Folgenden GER, siehe Trim et al. 2001, 2009), der inzwischen um das Companion Volume ergänzt $^{2}$ wurde (vgl. auch Nickenig in diesem Band), markiert in der Fremdsprachendidaktik einen Meilenstein. Neben seinen in den vergangenen Jahren immer wieder beschriebenen Funktionen im Bereich Niveaubeschreibungen, Kompetenzen, Strategien u.ä. ist vielleicht etwas in Vergessenheit geraten, was ebenfalls neu war: Die Situierung von Fremdsprachendidaktik in einen Kontext von Mehrsprachigkeit. Dies wird sehr deutlich in der Studie Die Umsetzung des Gemeinsamen europäischen Referen₹rabmens für Sprachen in den europä̈schen Bildungssystemen von 2013 (Broek \& van den Ende 2013). Der GER dient vorzugsweise der Standardisierung von Fremdsprachenkenntnissen, und damit der Vergleichbarkeit, er schafft eine Basis für Lehrpläne und Leitlinien, Lehrbücher und Prüfungen. Erst 2008 wurde durch die Empfehlung des Ministerkomitees an die Mitgliedstaaten der Fokus auf die Mehrsprachigkeit gelegt, obwohl diese im GER immer wieder thematisiert wird: So schon im ersten Satz des Vorwortes: „Mehrsprachigkeit und kulturelle Kompetenz sind die zentralen Themen dieser umfassenden Publikation zum Fremdsprachenlernen, [...]“ (GER: 3, Hervorhebungen im Original) und dann in einem eigenen Unterkapitel „Was bedeutet ,Mehrsprachigkeit'?“. Insgesamt 19-mal kommt der Begriff Mehrsprachigkeit im GER vor!

\footnotetext{
${ }^{3}$ Europa ist eigentlich politisch nur denkbar, wenn seine kulturelle Vielfalt, also auch die sprachliche Diversität berücksichtigt wird. Durch eine geeignete Didaktik für das Englische als lingua franca würde das Erlernen weiterer Fremdsprachen nicht ausgeschlossen und das Englische erschiene nicht per se als „Killersprache“.

${ }^{4}$ https://rm.coe.int/cefr-companion-volume-with-new-descriptors-2018/1680787989 [22.02.2019].
} 
Im Companion Volume kommt der Begriff plurilingualism 16-mal vor, der Begriff plurilingual sogar 126-mal! Dies zeigt deutlich die noch weiter gestiegene Wichtigkeit der Grundlage der Mehrsprachigkeit für den Referenzrahmen. Auch wenn man die faszinierenden Neuerungen wie die Darstellung von Mehrsprachigkeitsprofilen und Niveaubeschreibungen für Mehrsprachigkeitskompetenzen begrüßen muss, bleibt als Kritikpunkt immer noch der zentrale Blick auf das klassische Europa. Und ein weiterer Kritikpunkt wird auch durch die Neufassung nur unzureichend beantwortet: Bei aller Wichtigkeit von Mehrsprachigkeit gehen die Kompetenzbeschreibungen von einzelnen, isolierten Sprachen aus. Zwar werden sprachübergreifende Bereiche angesprochen, wie Mediation, Plurilingual und Pluricultural Repertoire, aber die Kernsubstanz der Beschreibungen der linguistischen Kompetenzen bezieht sich auf je eine Einzelsprache.

Einen grundlegend anderen Ansatz wählt hier der Referenzrabmen für plurale Ansätze zu Sprachen und Kulturen (REPA) (European Centre for Modern Languages 2019). Mit diesem Dokument ist aber keine Grundlagenkritik des GER verbunden, es begreift sich als Ergänzung zu den Instrumenten des Europarats, wie auf der entsprechenden Webseite ${ }^{5}$ deutlich gemacht wird. Damit können einige der angesprochenen Kritikpunkte des GER gelöst werden.

\section{Mehrsprachigkeit}

„Mehr“ bedeutet „mehr als zwei“, insofern ist die Zweisprachigkeit allenfalls als eine Spezialform, vielleicht sogar eine reduzierte Spezialform der Mehrsprachigkeit zu verstehen. Deswegen bezieht sich der Ausdruck individuelle Mehrsprachigkeit auf eine mindestens dreisprachige Persönlichkeit. Vielleicht ist es an dieser Stelle notwendig, darauf zu verweisen, dass eine mehrsprachige Persönlichkeit keineswegs ein Individuum ist, welches drei oder mehr Sprachen fließend spricht und/ oder schreibt! Diese Diskussion ist eigentlich schon lange abgeschlossen: Mehrsprachig ist eine Person, die in verschiedenen Sprachen ihren Alltag bewältigen kann (vgl. Lüdi \& Py 2009). Dies beinhaltet, dass sie Kompetenzen in verschiedenen Sprachen hat, die sie situationsadäquat einsetzt. So gibt es z.B. rezeptive oder domänenspezifische Mehrsprachigkeit (ich kann über bestimmte Themen in einer Sprache sprechen, über andere Themen in einer weiteren) und anderes.

Nun müssen wir uns aber in unserem Zusammenhang fragen, was eigentlich mit dem Begriff Mehrsprachigkeit gemeint ist, oder besser noch - seinerzeit gemeint war - und heute in der aktuellen Situation vielleicht anders interpretiert werden muss. Hat sich doch Europa in den bald zwanzig Jahren seit Erscheinen des GER stark verändert. Auch muss vorausgeschickt werden, dass es in diesem Artikel prinzipiell um individuelle Mehrsprachigkeit geht, nicht um territoriale oder institutionelle Mehrsprachigkeit.

\footnotetext{
${ }^{5}$ http://carap.ecml.at/ [23.01.2019].
} 


\section{1 „Klassische“ Mehrsprachigkeit - Bildungsmehrsprachigkeit}

Wenn im GER von Mehrsprachigkeit die Rede ist, auch im Companion Volume, so vor dem Hintergrund der europäischen Sprachensituation, also eines Europas mit den drei großen Sprachfamilien (Germanisch, Romanisch, Slavisch) und Finnisch, Estnisch, Ungarisch. Es war das Anliegen des Europarates und der Europäischen Union, diese europäische Sprachenvielfalt zu erhalten und die Bürger/-innen dazu zu befähigen, mit dieser Vielfalt umzugehen. Hier handelt es sich um die klassische Mehrsprachigkeit, die man auch als Bildungsmehrsprachigkeit bezeichnen kann. Durch das Barcelona-Prinzip sollte neben der Erstsprache eine allgemeine Verkehrssprache als erste Fremdsprache und eine weitere europäische Sprache als zweite Fremdsprache gelernt werden. ${ }^{6}$ Es darf hier aber nicht verschwiegen werden, dass den europäischen Anstrengungen in Bezug auf Mehrsprachigkeit etwas sehr Rhetorisches anhaftet: Die Mehrsprachigkeitsdebatte kreist um sehr wenige Sprachen und ignoriert die europäische Vielsprachigkeit (vgl. Krumm 2003: 36f.).

Die Studie von Kruse (2012) zeigt aber noch ein weiteres Phänomen auf, welches sich durch die Forderung L1 plus 2L (Erstsprache plus 2 Fremdsprachen) ergibt: Da inzwischen klar ist, dass eine der beiden Fremdsprachen Englisch ist, stellt sich die Frage, was die zweite sein soll. Hier gibt es die Tendenz, eine kleinere europäische Sprache zu lernen, was einer Absage an die großen europäischen Sprachen gleichkommt. Kruse formuliert klar und deutlich, dass die politische Diskussion um die europäische Mehrsprachigkeit auch von ,nationalen Interessen des Machterhalts und der Ausweitung des sprachlichen Einflusses“" (Kruse 2012: 4) geprägt ist. Auf der einen Seite führt die Sonderstellung des Englischen als lingua franca zu immensen Einnahmen der englischsprachigen Länder durch den großen Ausbau der Sprachlernangebote, auf der anderen Seite wehren sich die größeren Sprachgemeinschaften wie z.B. Frankreich (aber auch Deutschland, Italien und Spanien) durch politische und finanzielle Anstrengungen. Aber diese Konkurrenz schadet teilweise dem eigentlichen Anliegen der Mehrsprachigkeit. ${ }^{7}$

Die Diskussion ums Sprachenlernen in Europa bezieht sich in der klassischen Mehrsprachigkeitsdebatte auf die europäischen Sprachen, die als Schulsprachen unterrichtet werden, und dies sind inzwischen herzlich wenige. ${ }^{8}$ Außerdem wird in vielen sprachpolitischen Diskussionen immer noch implizit von monolingualen

\footnotetext{
${ }^{6}$ Es muss hier aber angemerkt werden, dass sich die Struktur mit einer allgemeinen Verkehrssprache (heute Englisch) und einer weiteren europäischen Sprache erst mit der Zeit herauskristallisiert hat. In den frühen Formulierungen von L1 plus 2L war wohl eher daran gedacht, dass durch die individuelle Dreisprachigkeit mit freier Sprachenwahl die allgemeinen Kommunikationsmöglichkeiten erweitert würden.

${ }^{7}$ Der Austritt von Grossbritannien aus der EU (Brexit) führt indessen zu einer Wiederaufnahme der Sprachenfrage. Ob dies aber eher eine rhetorische Frage ist, da schon so viele Festlegungen auf Englisch erfolgt sind, wird sich weisen. Auf jeden Fall ist es wohl delikat, zum Erlernen einer EUVerkehrssprache in Länder zu gehen, die nicht der EU angehören.
}

${ }^{8}$ Die EU umfasst derzeit 24 Amtssprachen, 5 halbamtliche Sprachen. 
Lernenden ausgegangen, obwohl schon lange Zeit eine deutliche Zunahme von bilingualen Schüler/-innen zu bemerken ist. Verschiedensprachliche Eltern haben durch die europäische Mobilität stark zugenommen.

\subsection{Aktuelle - migrationsbasierte - Mehrsprachigkeit}

Obwohl Migration schon ein länger diskutiertes Thema ist, wird erst in den letzten Jahren die Mehrsprachigkeit explizit wahrgenommen, die durch Migrationsbewegungen entstanden ist. Dies liegt wahrscheinlich daran, dass es ganz unterschiedliche Migrationsbewegungen gibt. So wurden die früheren „Wanderungsbewegungen" unter allen möglichen Aspekten betrachtet, selten jedoch hinsichtlich der Sprach(en)problematik. Wahrscheinlich erst durch die „Migrationswellen“ der letzten Jahre gerät nun auch die Sprachproblematik in den Fokus. Vielleicht auch deswegen, weil ja durch diese Wanderungen völlig andere Sprachen eine Rolle spielen als die miteinander verwandten europäischen Sprachen. Besonders brisant ist hier die Tatsache, dass (nicht nur in Deutschland oder der Schweiz) in der Vergangenheit angenommen wurde, Gastarbeiter/-innen und besonders Migranten/-innen brauchten ja nicht viel Sprache, da sie ja wieder in ihr Land zurück gehen (müssen).

In den Schulen finden sich zunehmend Lernende, die sogenannte Herkunftssprachen mitbringen, die aber im schulischen Unterricht sehr unterschiedlich bewertet und berücksichtigt werden. So entscheiden in Deutschland die Bundesländer, ob Unterricht der Herkunftssprachen an öffentlichen Schulen gegeben wird oder in ausserschulische Einrichtungen ausgelagert werden muss. Dementsprechend unterscheidet sich dann natürlich auch die Bewertung dieses Sprach- und Kulturunterrichts. Aber vielleicht sollte sich auch der schulische Fremdsprachenunterricht, der ja neueren Ansätzen entsprechend mehrsprachigkeitsdidaktisch unterlegt sein sollte, mit dieser Form von Mehrsprachigkeit auseinandersetzen. Diese Zielgruppe „mehrsprachige Migranten/-innen“ wird aber im GER (noch) nicht genannt (vgl. Schneider et al. 2017: 4).

Diese migrationsbedingte Mehrsprachigkeit bringt eine weitere Zunahme an Sprachen in Europa mit sich, die die gar nicht so heile Welt der klassischen Mehrsprachigkeitsdiskussion gehörig durcheinanderwirbelt (zu Überlegungen betreffend dieser neuen, migrationsbedingten Mehrsprachigkeit und ihrer Integration in die Bildungssysteme siehe Bundeszentrale für politische Bildung 2008, Erfa-Gruppe „Migration und Integration“ und Arbeitsgruppe „Sprachen“ EDK-Ost-Kantone und Fürstentum Liechtenstein 2007). 


\section{Zum Europäischen Sprachenportfolio}

Das Europäische Sprachenportfolio (im Folgenden ESP) ist eine Anwendung des GER und „wurde entwickelt, um das mehrsprachige und mehrkulturelle Repertoire jedes einzelnen Lernenden zu fördern“ (ESP 2010). Von 2000 bis 2010 wurden 118 ESP von der Validierungskommission des Europarats akkreditiert. Auf der Webseite des Europarats ${ }^{9}$ können Informationen zu diesen ESP analysiert werden (zur Kurzdarstellung der Funktionen des ESP siehe Langner 2011).

\subsection{Arten des Europäischen Sprachenportfolios (ESP)}

Seit seiner Einführung im Jahre 2000 (es war das Schweizer Sprachenportfolio (vgl. Schneider o.J.)) wurden in zahlreichen europäischen Ländern ESP eingeführt. Dabei wurden verschiedene Zielgruppen anvisiert, für deren unterschiedliche Bedürfnisse auch unterschiedliche Portfolios erstellt wurden.

\section{Portfolios für Kinder und Jugendliche}

Schon im Jahr 2000, also zeitgleich mit dem ersten akkreditierten Portfolio für Erwachsene, erschien in Frankreich das erste ESP für Kinder in der Primarschule - Altersgruppe 6-10 Jahre. Insgesamt wurden während der Akkreditierungszeit 38 ESP für die Zielgruppen von 3 bis 15 Jahren akkreditiert. Drei ESP sind für das Kindergarten-Niveau konzipiert, eine größere Anzahl zielt auf die Primarstufe, teilweise auf den Übergang von Primarstufe zur Sekundarstufe I (Altersgruppe 9 bis maximal 15 Jahre).

Die Schweiz z.B. hat das Portfolino (4 bis 7 Jahre) und das ESP I (7-11 Jahre). Eine Reihe von Ländern hat ESP für dieselben Altersgruppen (für Deutschland siehe Niedersächsisches Institut für frühkindliche Bildung und Entwicklung 2015).

\section{Portfolios für Erwachsene}

20 validierte ESP für Erwachsene finden sich auf der genannten Webseite. Aber auch hier werden unterschiedliche Zielgruppen angesprochen. So nennen acht ESP explizit als Zielgruppen Migranten/-innen und Sprecher/-innen von Minderheitssprachen. Aber auch ein Portfolio für Blinde und Sehbehinderte ist dabei (für erwachsene Migranten/-innen siehe Lazenby Simpson \& Barbara 2012).

Interessant sind hier die Südtiroler Sprachenportfolios, die (ähnlich wie die Schweizer Portfolios) weitere Landessprachen einschließen und für die älteren Lernenden dann auch Englisch (vgl. Gelmi o.J.).

9 https://www.ecml.at/Thematicareas/EvaluationandAssessment/EuropeanLanguagePortfolio/tab id/4179/Default.aspx [02.07.2019]. 


\section{Hocbschul-Sprachenportfolios}

Für die spezifischen Sprachbedürfnisse von Studierenden wurden ebenfalls ESP entwickelt, manchmal einfach durch Erweiterung der Kriterien um zusätzliche Beschreibungen, bisweilen aber auch durch ganz spezifische Kriterien für Studierende (vgl. Langner 2004). Neben dem Schweizer European Language Portfolio Higher Education und dem Cercles Portfolio, die beide schon 2002 validiert wurden, folgten solche in Albanien, den Niederlanden, Italien, Bulgarien, Portugal, Österreich und Ungarn.

Etwas, was die Schweizer Version des Hochschul-Portfolios auszeichnet, ist sein weitgehend selbsterklärender Aufbau. Für die einzelnen Bestandteile des ESP gibt es beispielhaft ausgefüllte Dokumente, die zeigen, wie man - auch ohne explizite Anleitung - mit dem Instrument arbeitet. Diese Idee haben leider nur wenige ESP aufgegriffen.

\section{Europäisches Portfolio für Sprachlehrende in Ausbildung (EPOS A)}

Ein Portfolio, welches den klassischen Weg des ESP dann schon teilweise wieder verlässt, ist das EPOSA/EPOSTL (vgl. Newby 2012), welches sich an zukünftige Sprachunterrichtende wendet. Diese müssen zwar auch in den später zu unterrichtenden Sprachen Kompetenzen aufweisen, zusätzlich aber auch eine ganze Reihe anderer Fähigkeiten. Bei diesem Portfolio werden meiner Meinung nach die ursprünglichen Funktionen betont: Die Begleitung von Lernprozessen, die Setzung von Lernzielen und die Dokumentation von erreichten Ergebnissen.

\subsection{Elektronische Versionen - ePortfolios}

Bei diesen ESP handelt es sich nicht um andere Arten, sondern um ein anderes Medium. Während die ursprünglichen ESP Papierversionen waren, die eine Reihe von negativen Begleiterscheinungen aufwiesen (Unhandlichkeit, Unübersichtlichkeit, mangelnde Selbsterklärung), versuchen die eESP genau diese Negativfaktoren zu verringern. Ganz besonders zeigt sich dies beim Dossier: Durch die Digitalisierung haben Lernende (und Lehrende) heute vielfältige Möglichkeiten, Lernergebnisse leicht $\mathrm{zu}$ sammeln und somit zu dokumentieren (Tonaufnahmen, Videoclips, geschriebene Dokumente) und diese auch sehr leicht zu aktualisieren.

Es gibt inzwischen eine große Anzahl solcher elektronischer Versionen, teilweise plattformbasiert und hier leider oft auch als proprietäre Lösungen, teilweise als einfache Webseiten konzipiert.

Problematisch an den proprietären Lösungen ist, dass ein Grundgedanke des ESP so nicht einfach zu realisieren ist: Das Portfolio ist Eigentum des Benutzers/der Benutzerin und sollte auf einfache Weise portabel sein! Diesen Gedanken greift eine elektronische Version auf, die von Maria Luisa Perez-Cavana, Bärbel Kühn und vom Autor dieses Textes mitentwickelt wird: EPOS. 


\subsection{Anwendung der Portfolios}

Obwohl das ESP schon 2000 eingeführt wurde, gestaltet sich seine Verwendung in den europäischen Ländern sehr unterschiedlich. Die vielen Portfolios in verschiedenen Arten für sehr unterschiedliche Zielgruppen sind die eine Seite, die vergleichsweise seltene Verwendung ist die andere. Und dies, obwohl praxis- und unterrichtsbezogene Ansätze und Materialien vorliegen (Hutterli 2007, Keller 2010). Dies hat unterschiedliche Gründe:

- Zum einen liegt das an der etwas einseitigen Ausrichtung an der Leistungsbewertung mit Hilfe der Kriterienraster. Die im ESP formulierten Kann-Beschreibungen basieren auf den jeweiligen Deskriptoren des GER, jedoch sind sie als „Ich kann-Beschreibungen“ formuliert. Obwohl diese ursprünglich für die Selbstevaluation gedacht waren, wurden diese Kriterien, vielleicht wegen ihrer leichten Handhabbarkeit, in den Vordergrund gerückt. Damit sind die linguistischen Kompetenzen, die zur Beschreibung von jeweiligen Sprachniveaus dienen sollten, einfach zur Bewertung gebraucht worden.

- Ein weiterer Grund ist sicher darin zu sehen, dass das ESP in seiner Papierform ein eher unhandliches Produkt war und bei seiner Einführung immer darauf hingewiesen wurde, dass es in seiner Gesamtheit verwendet werden solle! Ein Gebrauch von Teilen war nicht explizit vorgesehen. ${ }^{10}$

- Ein dritter Grund ist, dass das ESP nicht unbedingt selbsterklärend ist, es also in spezifischen Veranstaltungen eingeführt werden muss. Dies empfinden viele Lehrpersonen als Zeitverlust zu Lasten der eigentlichen, meist schon knappen, Lernzeit.

- Und der wahrscheinlich entscheidende Grund ist die Tatsache, dass mit dem ESP ein innovatives Instrument vorgestellt wurde, welches $\mathrm{zu}$ seiner sinnvollen Verwendung auch innovative Lernsituationen erfordert hätte. Dies wurde vielleicht nicht immer deutlich, und so wurde dieses innovative Dokument in traditionellen Lernsituationen eingesetzt. Die für eine sinnvolle Verwendung des ESP notwendige Diskussion von Autonomieförderung, von Lernreflexion, von Eigenverantwortung, von sinnvoller Lernbegleitung unterblieb. Es hätte das benötigt, was inzwischen unter dem Begriff von „Lernberatung“ auch an den Schulen angekommen ist.

\footnotetext{
${ }^{10}$ Eine interessante Erfahrung aus Japan auf einem Kongress zu autonomem Sprachenlernen 2007: Japanische Kollegen sagten ganz klar, dass ein Dokument grösser als das Format A5 in Japan zum Scheitern verurteilt wäre!
} 
Einen gewissen Ausweg boten dann die sogenannten ePortfolios. Aber auch hier erwies sich die häufige Komplexität der digitalen Lösungen als Hindernis für die Verwendung.

\subsection{Mehrsprachigkeitsaspekte}

Ähnlich wie auch für den Referenzrahmen wird bei Diskussionen um das ESP immer wieder die Förderung der Mehrsprachigkeit erwähnt. Auch die entsprechenden Webseiten (Europarat, ECML, Wikipedia etc.) verweisen - meist schon an prominenter Stelle ganz am Anfang - auf die Förderung von Mehrsprachigkeit. Es ist - die meisten ESP sind zwischen 2000 und 2008 entwickelt worden - historisch zumindest einigermaßen verstehbar, dass bei dieser wichtigen Ausrichtung an Mehrsprachigkeit die „klassische“, also Bildungsmehrsprachigkeit, im Vordergrund stand. Der Begriff „einigermaßen“ soll hier aufzeigen, dass das „Problemfeld“ Migration nicht erst im 21. Jahrhundert virulent wurde, es drang aber in der europäischen Sprachenpolitik nicht wirklich ins Bewusstsein (typisch für diesen Ansatz: das Europäisches Sprachenportfolio).

Wenn man die (europäische) Geschichte der Migration betrachtet (vgl. z.B. Bacci 2015 oder schon Brüggemann-Buck 1999), so wird schon in den 90er Jahren des letzten Jahrhunderts sehr deutlich, dass neue Wanderungsbewegungen zur europäischen Mobilität hinzutreten: Geflüchtete, Asylbewerber/-innen aus zunehmend nichteuropäischen Ländern und Kulturen der EU. Es wird eine ganze Reihe politischer Abkommen geschlossen, die eine gemeinsame Politik in Bezug auf diese Migration zum Ziel haben: Schengener Übereinkommen 1990, Vertrag von Maastricht 1993, Dublin-Abkommen 1997. Es ist schon bemerkenswert, wie die beiden wichtigsten europäischen Institutionen - Europäische Union als politische und Europarat als eher kulturpolitische Einrichtung - die damalige Wirklichkeit unterschiedlich wahrgenommen haben. Politisch realisiert man die außereuropäische Migration und reagiert darauf (nicht immer sehr gelungen), aber kulturpolitisch wird Mehrsprachigkeit meist nur im klassischen Sinn als Mehrsprachigkeit mit (einigen) europäischen Sprachen verstanden.

Dies ändert sich, zumindest auf der Ebene von Richtlinien ab 2008 mit der Publikation von Beacco - The role of languages in policies for the integration of adult migrants. Und noch deutlicher wird es im Dokument des Europarates von 2014, The linguistic integration of adult migrants, in dem klar darauf hingewiesen wird, dass der Referenzrahmen teilweise missbraucht wird, um Geflüchteten etc. durch „objektive" Tests zu zeigen, dass sie nicht die erforderlichen sprachlichen Bedingungen des Ziellandes erreicht haben!

In diesem letztgenannten Dokument ist an einer Stelle vom ESP die Rede: Relativ weit hinten im Kapitel Teaching methodology zum Stichwort lebenslanges Lernen heißt es: „One of the purposes of the European Language Portfolio is to support the development of such skills, which may help to explain why it has been particularly successful when used with adult migrants" (Council of Europe 2014: 38). 
Migranten/-innen sind wohl die ideale Gruppe der lebenslang Lernenden! Und dann noch ein kurzes Kapitel zum Thema The European Language Portfolio as an assessment instrument, in dem aber ganz deutlich formuliert wird, dass das ESP ein Instrument für Lernende ist, die damit ihre Lernziele bestimmen und ihre Lernergebnisse selbst bewerten können.

In unserem Zusammenhang noch interessanter ist das Dokument von Beacco et al. (2017). Aber gerade mit diesem Dokument wird deutlich, dass in der neueren Diskussion der europäischen Institutionen teilweise noch der Referenzrahmen erwähnt wird, aber die Nutzung des ESP ziemlich aus dem Blick geraten ist. Vielleicht liegt dies auch daran, dass trotz der häufigen Erwähnung der Mehrsprachigkeitsförderung das ESP in seiner Anlage doch eine eher additive Mehrsprachigkeit fördert: Die Dokumente sind einzelsprachlich entworfen und selbst im Dossier werden die Beispiele von best practice nebeneinander abgespeichert. Es fehlt hier die Umsetzung von (zugegebenermaßen sehr jungen) Beispielen für mehrsprachige Kompetenzen (siehe Forschungsgruppe „Dynamik mehrsprachiger Kompetenzen")!

Aber dieses Dokument zeigt auch etwas anderes sehr deutlich: grundlegende theoretische Divergenzen. Wenn im ersten Beitrag von Ofelia García ${ }^{11}$ ein revolutionär neuer theoretischer Blick aufscheint, der die bisherige linguistische Grundlegung der Spracherwerbstheorien aus den Angeln hebt, so bleiben die meisten anderen Beiträge dieses Bandes der klassischen Sichtweise verhaftet. García wendet sich deutlich ab von der klassischen Sichtweise von Einzelsprachen - für sie gibt es diese nicht, sondern nur das Sprechen des Individuums. Dies ist eine klare Absage an die Theorien der balancierten Zwei- oder Mehrsprachigkeit, also eine Auffassung der Mehrsprachigkeit aus Sicht der Monolingualen. ${ }^{22}$ Das Individuum (hier spezifisch der/die Migrant/-in) benutzt unterschiedliche sprachliche Eigenschaften aus verschiedenen Sprachen, es ist also mehrsprachig in einem neuen Sinn. Das mehrsprachige Individuum „versprachlicht“ (languaging = versprachlichen) also seine Kommunikationsbedürfnisse. Wenn man diesen Ansatz ernst nimmt, ergeben sich große Konsequenzen für den Sprachunterricht und für das Testen. Nur im Beitrag von Laimer und Wurzenrainer im Band von Beacco et al. (2017) wird dieser Ansatz aufgegriffen, alle weiteren, durchaus interessanten Beiträge dieses Bandes bleiben dem klassischen Ansatz von Einzelsprachen, Nationalsprachen, Zweit- und Fremdsprachen verhaftet.

\footnotetext{
11 Der Ansatz von Garcia stammt selbstverständlich nicht aus dem Jahr 2017, sondern ist schon einige Jahre älter und hat sich in der Soziolinguistik inzwischen auch etabliert. Dies verhindert aber nicht, dass in der Mehrsprachigkeitsdidaktik erst langsam in diese Richtung gedacht wird.

12 In dieser Sicht bedeutet z.B. Zweisprachigkeit ein doppeltes hundertprozentiges Sprachvermögen. Demnach addiert man z.B. den Wortschatz und muss feststellen, dass bei Zweisprachigen eben kein Wortschatz zu 200\% vorhanden ist. Da funktionale Aspekte der Mehrsprachigkeit ausgeklammert werden, ist es eher eine Defizitsicht auf Mehrsprachigkeit.
} 


\section{Ausblick}

Kehren wir zurück zu unserem Ausgangspunkt: Portfolio und Mehrsprachigkeitsförderung. Es ist leider nicht so gekommen, wie sich die Urheber des ESP dies gedacht haben. Die aktuelle Diskussion, ausgelöst durch die starken Migrationsbewegungen der letzten Jahre, läuft momentan ziemlich an der Portfolioarbeit vorbei, obwohl einzelne Diskussionsbeiträge der letzten Jahre auch für die Spracharbeit mit Geflüchteten das ESP immer mal wieder erwähnen.

Um eine eventuelle Renaissance dieses eigentlich wichtigen und sinnvollen Werkzeugs zu ermöglichen, müssen wir vielleicht von den eingefahrenen Wegen Abschied nehmen (vgl. hierzu Kühn et al. 2014):

- Das ESP ist kein monolithischer Block. Es sollte normal sein, dass man bestimmte Teile des ESP auch ohne den Gesamtzusammenhang verwenden kann und darf. Warum z.B. dem/der Lernenden nach der Selbsteinschätzung mit dem Grobraster nicht einfach nur die jeweilig passenden Kriterienraster geben? Also „Zusammenhängend Sprechen = B1 - Raster A2 und B2“. Und vielleicht das Dossier prinzipiell digital aufbauen und führen lassen.

- Vielleicht müssten manche der Dokumente auch überarbeitet werden. Ein Grundvorwurf an das ESP besteht ja darin, dass es zwar die Mehrsprachigkeit fördern soll, aber von seiner Grundkonstruktion her eine additive Mehrsprachigkeit fördert. Die Mehrsprachigkeitsdiskussion verabschiedet sich ja langsam von den Kategorien additiver und subtraktiver Mehrsprachigkeit. Und um eine integrative Mehrsprachigkeit zu fördern, müssten wohl die Dokumente anders aufgebaut werden.

- Das ESP hat verschiedene Funktionen, ist also nicht auf die Evaluationsfunktion begrenzt. Diese ist vielleicht auch nicht die wichtigste Funktion. Lernbegleitung und -dokumentation sind gerade für Lernprozesse von Erwachsenen eminent wichtig.

- Ein innovatives Instrument kann nicht einfach auf traditionelle Strukturen aufgesetzt werden. Aber genau dies wurde meistens gemacht. Da inzwischen die früher nur auf Hochschul-Niveau diskutierte (Sprach-) Lernberatung in der Erwachsenenbildung und in den Schulen angekommen ist, könnte hier eine bessere Grundlage für die neue Verwendung des ESP geschaffen werden (vgl. Kühn \& Langner 2011). Gerade auch im Bereich der Spracharbeit mit Migranten/-innen wird Lernberatung intensiv diskutiert. So habe ich Ende 2017 für eine Zürcher Institution im Bereich der Arbeit mit Geflüchteten und Asylbewerber/-innen Konzepte von Lernberatung vorgestellt. 
- Und was verschiedene Länder inzwischen schon tun, sollte generell überlegt werden: Ist die Papierversion mit ihrer Schwerfälligkeit noch zeitgemäß? Bräuchte es nicht digitale Versionen? Wobei wir hier mindestens zwei grundlegende unterschiedliche Formen von eESP unterscheiden müssen:

- Die einfache Übertragung der Papierversionen in elektronische Dokumente und die damit verbundene leichtere Handhabbarkeit, besonders auch der wichtigen Funktion des Dossiers.

○ Die Neukonzeption einer Plattformlösung, wie sie in mehreren Beiträgen dieses Bandes zu EPOS diskutiert wird. Hierbei müsste aber meines Erachtens der Modulgedanke noch stärker entwickelt werden: die einzelnen Bestandteile des ESP (und vielleicht auch weitere ergänzende Dokumente) sollten als eigenständige Module konzipiert werden, die unabhängig voneinander eingesetzt werden könnten. So könnte sich jede/r Lernende die für ihn/sie wichtigen Komponenten/Module zu seinem/ihrem Portfolio zusammenfügen!

○ Durch eine solche elektronische Plattformlösung könnten dann z.B. auch verschiedene Teile unterschiedlicher Portfolios oder Referenzrahmen integriert werden: Die grundlegende Schwäche des GER durch seine Orientierung an Einzelsprachen könnte beispielsweise durch die Hinzunahme von Teilen des REPA ,behoben" werden.

○ Und vielleicht wäre es am besten, eine Hybridversion zu erstellen, in der einzelne Teile auch als Papierdokumente eingesetzt werden könnten - sie liegen dann als druckbare PDF-Dokumente vor. ${ }^{13}$

- Dies ist nicht das einfache Mitschwimmen auf dem derzeitigen Hype der Digitalisierung, sondern der Versuch, die Vorteile von elektronischen Portfolios zu nutzen.

Vielleicht können wir mit solchen Ideen einem guten und wichtigen Lerninstrument nach über zwanzig Jahren zum Durchbruch verhelfen, und damit nicht nur, aber auch die Mehrsprachigkeit in Europa fördern. Vielleicht klappt dies nicht mehr wirklich für die europäische Mehrsprachigkeit (L1 plus 2L), die vielleicht doch nur für eine Elite erreichbar ist, aber eben für die auch sprachlichen Herausforderungen, die uns die Migrationsbewegungen stellen. Einen Versuch, das ESP in einen leicht utopischen Ansatz zu integrieren, bieten Kühn und Langner (2015).

\footnotetext{
13 Wir haben derzeit ja eine gewisse Rückwendung zum Analogen: Gerade die sogenannte Generation $Z$ (Scholz 2014), die derzeit 16-18-Jährigen, beginnt sich mit „Greifbarem“ auseinanderzusetzen und erkennt z.B. gewisse Lernvorteile des Arbeitens mit Papier (Sax 2017).
} 


\section{Literatur}

Bacci, Massimo Livi (2015): Kurze Geschichte der Migration. Berlin: Wagenbach.

Beacco, Jean-Claude (2008): The Role of Languages in Policies for the Integration of Adult Migrants. Strasbourg: COE. https://rm.coe.int/16802fc0b0 [01.03.2019].

Beacco, Jean-Claude; Krumm, Hans-Jürgen; Little, David (2017): The Linguistic Integration of Adult Migrants. Some Lessons from Research. https:/ / www.degruyter. $\mathrm{com} /$ view/product/472830 [01.03.2019].

Broek, Simon; van den Ende, Inge (2013): Die Umsetzung des Gemeinsamen europäischen Referenærahmens für Sprachen in den europäischen Bildungssystemen. Brüssel: Europäische Union. http://www.europarl.europa.eu/RegData/etudes/etudes/ join/2013/495871/IPOL-CULT_ET\%282013\%29495871_DE.pdf [26.06.2019].

Brüggemann-Buck, Ilka (1999): Migration in Europa. Wanderungsbewegungen und ibre politischen Auswirkungen im Rabmen der Europäischen Integration. Marburg: Inauguraldissertation. http://archiv.ub.uni-marburg.de/diss/z2001/0406/ pdf/dibb.pdf [01.03.2019].

Bundeszentrale für politische Bildung (2008): Grundlagendossier Migration. http://www.bpb.de/gesellschaft/migration/dossier-migration/ [02.07.2019].

Council of Europe (2014): The Linguistic Integration of Adult Migrants. From One Country to Another, from One Language to Another. Strasbourg Cedex: Council of Europe Publications. https://rm.coe.int/16802fd54a [04.07.2019].

Council of Europe (2018): Common European Framework of Reference for languages: Learning, Teaching, Assessment. Companion volume with new descriptors. Strasbourg: Council of Europe. https:/ / rm.coe.int/cefr-companion-volume-with-newdescriptors-2018/1680787989 [22.02.2019].

Council of Europe (o.J.): European Languane Portfolio. Home. https://www.coe.int/ en/web/portfolio/home [02.07.2019].

Erfa-Gruppe „Migration und Integration“ und Arbeitsgruppe „Sprachen“ EDKOst-Kantone und Fürstentum Liechtenstein (2007): Sprachförderung für eine mehrsprachige Schülerschaft. Empfehlungen an die Kantone. Zürich: Bildungsdirektion. https://www.ar.ch/fileadmin/user_upload/Departement_Bildung_Kultur/ Amt_fuer_Volksschule/Foerderangebote/Sprachfoerderung_und_Mehrsprach igkeit.pdf [01.03.2019].

Europäisches Sprachenportfolio (2010): Mehrsprachigkeit und Gemeinsamer Europäischer Referenzrahmen. http:/ / www.sprachenportfolio.ch/page/content/ index. asp?MenuID=2082\&ID=3367\&Menu=14\&Item=1.2.2 [27.06.2019].

Europäisches Sprachenportfolio (o.J.): Profil der Sprachenkenntnisse. In fünf Schritten zu Ihrem Mehrsprachigkeitsprofil. http://www.sprachenportfolio.ch/page/content/ index.asp?MenuID=2051\&ID=3284\&Menu=14\&Item=7.2.2.2.1 [27.06.2019]. 
European Centre for Modern Languages (2011): Using the European Language Portfolio. http://elp.ecml.at/Portfolios/tabid/2370/language/enGB/Default.aspx [02.07.2019].

European Centre for Modern Languages (2019): Referenz̧ahmen für plurale Ansätz̧e zu Sprachen und Kulturen. http://carap.ecml.at/ [01.03.2019].

European Parliament (2019): Fact Sheets of the European Union. http:/ /www.europarl.europa.eu/atyourservice/de/displayFtu.html?ftuId=FT U_5.13.6.html [01.03.2019].

Forschungsgruppe Dynamik Mehrsprachige Kompetenzen (o.J.): Forschung. https://www3.unifr.ch/pluriling/de/forschung/dynamik-mehrsprachigekompetenz.html [01.03.2019].

García, Ofelia (2017): Problematizing linguistic integration of migrants: the role of translanguaging and language teachers. In: Beacco, Jean-Claude; Krumm, Hans-Jürgen; Little, David; Thalgott, Philia (Hrsg.): The Linguistic Integration of Adult Migrants. Some Lessons from Research. L'intergration linguistique des migrants adultes. Les enseignements de la recherche. Berlin, Boston: de Gruyter, 11-26.

Gelmi, Rita (o.J.): Südtiroler Sprachenportfolios. http:/ /www.provinz.bz.it/bildungsprache/didaktik-beratung/sprachenportfolio-esp.asp [27.06.2019].

Hufeisen, Britta; Neuner, Gerhard (Hrsg.) (2003): MehrsprachigkeitskonzeptTertiärsprachenlernen - Deutsch nach Englisch. Strasbourg: Council of Europe Publishing.

Hutterli, Sandra (2007): Europäisches Sprachenportfolio. Stufenübergreifendes, plurilinguales Lernen im europäischen Kontext. In: PH | Akzente 1, 7-10.

Keller, Marlies (2010): Unterrichtsmaterialien und-ideen für die Arbeit mit dem Europäischen Sprachenportfolio. Zürich: Pädagogische Hochschule.

Krumm, Hans-Jürgen (2003): Sprachenpolitik und Mehrsprachigkeit. In: Hufeisen, Britta; Neuner, Gerhard (Hrsg.): Mehrsprachigkeitskonzept-Tertiärsprachenlernen Deutsch nach Englisch. Strasbourg: Council of Europe Publishing, 35-49.

Kruse, Jan (2012): Das Barcelona-Prinzip: Die Dreisprachigkeit aller Europäer als sprachenpolitisches Ziel der EU. Frankfurt/M.: Lang.

Kühn, Bärbel; Langner, Michael (2011): Lernberatung, digitale Medien und Europäisches Sprachen-Portfolio. In: Vogler, Stefanie; Hoffmann, Sabine (Hrsg.): Sprachlernberatung für DaF. Berlin: Frank \& Timme, 93-105.

Kühn, Bärbel; Langner, Michael (2015): Schwarm-X: Die große Erzählung vom Europäischen Sprachenportfolio an der Schwelle zur post-digitalen Welt. In: Böcker, Jessica; Stauch, Anette (Hrsg.): Konzepte aus der SprachlehrforschungImpulse für die Praxis (Festschrift für Karin Kleppin). Frankfurt/M.: Lang, 125-149. 
Kühn, Bärbel; Langner, Michael; Perez-Cavana, Maria Luisa (2014): Zur bildungspolitischen und pädagogischen Aktualität von Lernberatung mit EPOS, der Online-Plattform des Europäischen Sprachenportfolios - Oder:

Tempora mutantur et nos mutamur in illis. In: Berndt, Annette; Deutschmann, Ruth-Ulrike (Hrsg.): Sprachlernberatung - Sprachlerncoaching. Frankfurt/M.: Lang, 213-234.

Laimer, Thomas; Wurzenrainer, Martin (2017): Multilingualism as a resource for basic education with young migrants. In: Beacco, Jean-Claude; Krumm, HansJürgen; Little, David; Thalgott, Philia (Hrsg.): The Linguistic Integration of Adult Migrants. Some Lessons from Research. L'intergration linguistique des migrants adultes. Les enseignements de la recherche. Berlin, Boston: de Gruyter, 117-122.

Langner, Michael (2004): Europäisches Sprachenportfolio Bereich Hochschule. In: Duricová, Alena; Hanuljaková, Helena (Hrsg.): Europäische Sprachenvielfalt Chance oder Herausforderung für die deutsche Sprache. Beiträge der VII. Tagung des Verbandes Deutschlehrer und Germanisten der Slowakei. Banská Bystrica: Pedagogická fakulta Univerzity Mateja Bela v Banskej Bystrici, 182-186.

Langner, Michael (2011): Das Europäische Sprachen-Portfolio. In: Fremdsprache Deutsch. Zeitschrift für die Praxis des Deutschunterrichts. Themenheft: Portfolioarbeit 45/2, 12.

Lazenby Simpson, Barbara (2012): European Language Portfolio for Adult Migrant Learning the Language of the Host Country. A Guide for Teachers. Strasbourg: Council of Europe.

Lüdi, Georges; Py, Bernard (2009): To be or not to be ... a plurilingual speaker. In: International Journal of Multilingualism 6/2, 154-167.

Newby, David (2012): Insights into the European Portfolio for Student Teachers of Languages (EPOSTL). Newcastle upon Tyne: Cambridge Scholars Publishing.

Niedersächsisches Institut für frühkindliche Bildung und Entwicklung (2015): Mehrsprachigkeit fördern: Europäisches Sprachenportfolio. https://www.nifbe.de/ component $/$ themensammlung? view $=$ item\&id $=544$ :mehrsprachigkeitfoerdern-europaeisches-sprachenportfolio\&catid=292 [01.03.2019].

Sax, David (2017): Die Rache des Analogen. Warum wir uns nach realen Dingen sebnen. Salzburg, Wien: Residenz.

Schneider, Günther (o.J.): Wozu ein Sprachenportfolio? Funktionen und Merkmale des Europäischen Sprachenportfolios (Schweizer Version). http:/ /www.unifr.ch/ids/ Portfolio/html-texte/teill-aufsatz-gu-sprachenportfolio.htm [26.06.2019].

Schneider, Günther; Lenz, Peter; Foster Vosicki, Brigitte u.a (2017): Gemeinsamer europäischer Referenzrabmen für Sprachen (GER) und Europäisches Sprachenportfolio (ESP). Bericht der SIG Arbeitsgruppe 3.1. Freiburg, Fribourg: IDT 2017.

Scholz, Christian (2014): Generation Z. Wie sie tickt, was sie verändert und warum sie uns alle ansteck.t. Weinheim: Wiley-VCH. 
Trim, John; Quetz, Jürgen; Schieß, Raimund; Schneider, Günther (Hrsg.) (2001/2009): Gemeinsamer europä̈scher Referenzrahmen für Sprachen. Lernen, lehren, beurteilen. Niveau A1, A2, B1, B2, C1, C2. Goethe-Institut Inter Nationes; Ständige Konferenz der Kultusminister der Länder in der Bundesrepublik Deutschland; Österreich. [Nachdr.]. Berlin: Langenscheidt. 